\title{
Malaria rapid diagnostic tests: Plasmodium falciparum infections with high parasite densities may generate false positive Plasmodium vivax pLDH lines
}

Jessica Maltha*1, Philippe Gillet², Lieselotte Cnops², Jef van den Ende², Marjan van Esbroeck² and Jan Jacobs 1,2

\begin{abstract}
Background: Most malaria rapid diagnostic tests (RDTs) detect Plasmodium falciparum and an antigen common to the four species. Plasmodium vivax-specific RDTs target $P$. vivax-specific parasite lactate dehydrogenase (Pv-pLDH). Previous observations of false positive Pv-pLDH test lines in P. falciparum samples incited to the present study, which assessed $P$. vivax-specific RDTs for the occurrence of false positive Pv-pLDH lines in P. falciparum samples.

Methods: Nine P. vivax-specific RDTs were tested with 85 P. falciparum samples of high ( $\geq 2 \%)$ parasite density. Mixed $P$. falciparum/P. vivax infections were ruled out by real-time PCR. The RDTs included two-band (detecting Pv-pLDH), threeband (detecting P. falciparum-antigen and Pv-pLDH) and four-band RDTs (detecting P. falciparum, Pv-pLDH and panpLDH).

Results: False positive Pv-pLDH lines were observed in 6/9 RDTs (including two- three- and four-band RDTs). They occurred in the individual RDT brands at frequencies ranging from $8.2 \%$ to $29.1 \%$. For 19/85 samples, at least two RDT brands generated a false positive Pv-pLDH line. Sixteen of 85 (18.8\%) false positive lines were of medium or strong line intensity. There was no significant relation between false positive results and parasite density or geographic origin of the samples.

Conclusion: False positive Pv-pLDH lines in P. falciparum samples with high parasite density occurred in 6/9 P. vivaxspecific RDTs. This is of concern as $P$. falciparum and $P$. vivax are co-circulating in many regions. The diagnosis of lifethreatening $P$. falciparum malaria may be missed (two-band Pv-pLDH RDT), or the patient may be treated incorrectly with primaquine (three- or four-band RDTs).
\end{abstract}

\section{Background}

Malaria rapid diagnostic tests (RDTs) are immunochromatographic tests targeting antigens of one or more Plasmodium species. Signals are visible as cherry-red to purple coloured lines, comprising a control line (which indicates that the test has been performed well) and one or two test lines. The initially developed two band tests generate a test line that targets $P$. falciparum by detecting either histidine-rich protein 2 (HRP-2) or P. falciparumspecific parasite lactate dehydrogenase (Pf-pLDH). The later developed three band tests include a second target

* Correspondence: j.maltha@student.maastrichtuniversity.nl

1 Faculty of Health, Medicine and Life Sciences (FHML), Maastricht, The Netherlands

Full list of author information is available at the end of the article that is common to the four Plasmodium species, such as aldolase or pan-specific parasite lactate dehydrogenase (pan-pLDH). However, the conventional three-band RDTs, detecting a $P$. falciparum-specific antigen and a pan-Plasmodium antigen, cannot distinguish between a $P$. falciparum infection and a mixed infection with $P$. vivax when both test lines are observed. Differentiation between the non-falciparum species is neither possible. $P$. falciparum and $P$. vivax infections require different treatment, which makes discrimination between the two species important. RDTs specific to $P$. vivax could be useful. There are two-band RDTs that detect Plasmodium vivaxspecific pLDH (Pv-pLDH), three-band RDTs in which Pv-pLDH is combined with HRP-2 or Pf-pLDH, and so- 
called four-band tests that combine detection of HRP-2, pan-pLDH and Pv-pLDH. The Pv-pLDH tests have undergone limited evaluation [1-3].

In previous evaluations of RDTs targeting $\mathrm{Pv}-\mathrm{pLDH}$, rare but consistent false positive $\mathrm{Pv}$-pLDH test lines were observed among P. falciparum samples, especially at high parasite densities [2-4]. These observations are of concern as this limits the potential use of the Pv-pLDH directed RDTs, both in endemic and non-endemic settings. In order to study the extent of this phenomenon among other RDTs, the present study was undertaken to challenge available RDT brands with a Pv-pLDH test line to a panel of $P$. falciparum samples with high parasite densities.

\section{Methods}

\section{Study design}

Several RDTs were retrospectively evaluated in a reference laboratory on a panel of stored whole blood samples obtained in patients suspected of malaria. The reference method was microscopy corrected by polymerase chain reaction (PCR).

\section{Patient samples}

In this study stored whole blood samples $\left(-70^{\circ} \mathrm{C}\right)$ were used, obtained in returned international travelers suspected of malaria presenting at the outpatient department of the Institute of Tropical Medicine (ITM), Antwerp, Belgium, or submitted by other Belgian laboratories to ITM in its function of National Reference Center. Samples had been obtained between 1996 and 2009 and were classified in regions of travel destinations according to the United Nations classification of geographical region and composition [5]. All samples were evaluated by microscopy and real-time PCR for species identification (ruling out mixed infections) and determination of parasite density, as described previously [6,7]. For the purpose of this study, the more convenient parasite density expressed by $\%$ of infected red blood cells was applied, thereby assuming $50,000 / \mu$ lo be equal to $1 \%$ of red blood cells parasitized [8]. P. falciparum-infected samples with parasite densities $\geq 2 \%(\geq 100,000 / \mu \mathrm{l})$ were selected.

\section{Malaria rapid diagnostic tests}

RDTs containing a $P$. vivax-specific test line were selected, including those published on the World Health Organization (WHO) list of RDT manufacturers with adequate evidence of good manufacturing [9] as well as others available on the international market. We checked the package inserts to ensure the antigen used was $P$. vivax-specific. In line with other comparative evaluations $[7,10]$ it was decided not to display individual RDT brand names because of the wide lot-to-lot variability and the frequent changes in composition and brand names and types [1].

\section{Test procedures}

Tests were performed according to the manufacturers' instructions, except that a pipette (Finnpipette, Helsinki, Finland) was used instead of the transfer devices supplied by the manufacturer. The laboratory technicians involved in the study have received a detailed training and their performance and agreement are monitored by participation to internal and external quality control assessments. Readings were done by three subsequent observers, of whom the first always was the one performing the test, and carried out at daylight assisted by an electric bulb. The observers were blinded to each others readings and to the results of microscopy and PCR. In case no control line was observed the test was considered invalid and repeated. Test line intensities were scored according to a system of five categories as described previously [6]: none (no line visible), faint (barely visible line), weak (paler than the control line), medium (equal to the control line) and strong (stronger than the control line). Test results were based on consensus agreement: the same test result observed by at least two out of three readers. In case of no consensus the result of the first reader was considered. Pv-pLDH lines generated by $P$. falciparum samples will be further referred to as false positive Pv-pLDH lines.

\section{Statistical analysis}

The nonparametric Spearman's rank correlation coefficient $r_{s}$ was used to measure the strength of association between parasite density and the number of RDTs with a false positive Pv-pLDH test line. Associations were considered significant at a $\mathrm{p}$-value $<0.05$. Inter-observer agreement for line intensities and positive and negative test results were expressed by kappa values for each pair of observers and by the percentage of overall agreement between the three observers.

\section{Ethical review}

The study was reviewed and approved by the Institutional Review Board of ITM and by the Ethical Committee of Antwerp University, Belgium.

\section{Results}

\section{Collection of samples and RDTs}

Eighty-five $P$. falciparum samples with a parasite density $\geq 2 \%(100,000 / \mu \mathrm{l})$ were selected. The parasite densities ranged from $2-35 \%$, with 30 samples of at least $20 \%$ parasite density.

The male:female ratio was 3:1, with a median age of 40 years (range 3-98 years) and three children were under the age of five years. Samples were obtained in West Africa $(\mathrm{n}=34)$, Middle Africa $(\mathrm{n}=22)$, East Africa $(\mathrm{n}=$ 
5), Southern Africa ( $n=4)$ and West Asia ( $n=1)$. Of 19 samples no data on the geographic origin were known and could not be retrieved. Four RDT brands (numbers. 1, 4, 7 and 9) were tested with less than 85 samples, due to either a lack of RDTs or a lack of sample.

Twenty different RDT brands were selected. Although their product name referred to $P$. vivax-specificity (for instance, by adding the epithet "Pf/Pv"), two of them in fact targeted pan-pLDH instead of Pv-pLDH: these RDTs were not considered for evaluation. Seven companies marketing eight RDT brands did not reply to the order of RDTs, despite several reminders via email contact. One $P$. vivax-specific three-band RDT was not included in this study because of bad clearance of the background, which made reading results impossible. The final panel consisted of nine different RDT brands from seven manufacturers, including one two-band (single Pv-pLDH test line), three three-band (Pv-pLDH and HRP-2 test line) and five four-band RDTs (Pv-pLDH, HRP-2 and panpLDH test line). Five RDTs had CE mark compliance and two were included in the WHO list of good manufacturing practices.

Three different four-band RDT brands (numbers 6, 7 and 8 ) had similar package inserts and the cassettes had identical morphology. Two three-band RDTs (numbers. 2 and 3) also had identical cassettes and similar package inserts. RDT numbers 1 and 4 were from the same manufacturer, as well as RDT numbers 2 and 5.

\section{Test characteristics}

There were no invalid test results. As expected, all the $P$. falciparum samples showed positive test lines for the HRP-2 and the pan-pLDH test lines, if present on the cassette. Table 1 lists the results for the Pv-pLDH test lines, expressed by line intensities. In total there were 85 false positive Pv-pLDH lines in six RDT brands, caused by a total of 42 samples. In the individual RDT brands they occurred at frequencies of $8.2 \%$ (7/85 samples) up to $29.1 \%$ (23/79 samples), among two-, three- and four-band tests. There was no difference between RDTs that were CE-marked or WHO-listed and those which were not.

Table 2 lists the details of consensus readings of line intensities for the false positive Pv-pLDH test lines, according to parasite densities and region of infection. In $2 / 736$ readings there was no consensus and the results of the first reader were considered. Nineteen samples generated a false positive line in at least two RDTs. There was no apparent relation between parasite density of samples and the occurrence of false positive $\mathrm{Pv}$-pLDH lines $\left(r_{\mathrm{s}}=\right.$ 0,155, $\mathrm{p}=0,153)$. False-positive Pv-pLDH lines occurred exclusively in samples from patients returning from Middle or West Africa (Table 3), but there was no significant relation between geographic origin of samples and falsepositive results.

Most (69/85, 81.2\%) false-positive Pv-pLDH readings were faint or weak (Table 1), but strong line intensities were observed in two RDT brands (Tables 1 and 2). Taken all readings together, inter-observer agreements for line intensity readings were good for HRP-2, pan-pLDH and Pv-pLDH test lines. For the Pv-pLDH line, in terms of positive and negative readings for all brands together, kappa values between pairs of observers were good $(0.79$, $0.70,0.77)$ and overall agreement was excellent $(88.0 \%)$. For the RDT brands considered separately, overall agreement for the $\mathrm{Pv}$-pLDH line ranged from $75.9 \%$ to $100 \%$.

\section{Discussion}

In this study, six out of nine $P$. vivax-specific RDTs showed false positive Pv-pLDH lines when challenged to a panel of $85 P$. falciparum samples with high $(\geq 2 \%)$ parasite densities, in which mixed infections with $P$. vivax

Table 1: Line intensity reading for Pv-pLDH lines in $P$. falciparum samples with parasite densities $\geq 2 \%$

\begin{tabular}{|c|c|c|c|c|c|c|c|}
\hline \multirow[b]{2}{*}{$\mathbf{N r}$} & \multirow[b]{2}{*}{ Type } & \multirow[b]{2}{*}{ Nr of samples tested } & \multicolumn{4}{|c|}{ Line intensity readings, number of samples } & \multirow[b]{2}{*}{ Total positive ( $\%$ of total samples) } \\
\hline & & & Faint & Weak & Medium & Strong & \\
\hline 1 & Two-band & 84 & 7 & 4 & 1 & 4 & $16(19.0 \%)$ \\
\hline 2 & Three-band & 85 & & & & & 0 \\
\hline 3 & Three-band & 85 & & & & & 0 \\
\hline 4 & Three-band & 66 & 2 & & 5 & 4 & $11(16.7 \%)$ \\
\hline 5 & Four-band & 85 & & & & & 0 \\
\hline 6 & Four-band & 85 & 8 & 13 & & & $21(24.7 \%)$ \\
\hline 7 & Four-band & 79 & 19 & 3 & 1 & & $23(29.1 \%)$ \\
\hline 8 & Four-band & 85 & 2 & 5 & & & $7(8.2 \%)$ \\
\hline 9 & Four-band & 82 & 1 & 5 & 1 & & $7(8.5 \%)$ \\
\hline
\end{tabular}

Two-band: Pv-pLDH test line; Three-band: Pv-pLDH and HRP-2 test line; Four-band: Pv-pLDH, HRP-2 and pan-pLDH test line. 
Table 2: False positive Pv-pLDH lines in 42 P. falciparum samples with parasite densities $\geq 2 \%$

\begin{tabular}{|c|c|c|c|c|c|c|c|c|c|}
\hline \multicolumn{3}{|c|}{ Samples } & \multicolumn{6}{|c|}{ RDT number and type } & \multirow[b]{2}{*}{$\begin{array}{c}\text { Total RDTs } \\
\text { positive }\end{array}$} \\
\hline $\mathbf{N r}$ & $\begin{array}{l}\% \text { parasite } \\
\text { density }\end{array}$ & Origin & 1. Two-band & 4. Three-band & 6. Four-band & 7. Four-band & 8. Four-band & 9. Four-band & \\
\hline 1 & 2,0 & MAF & - & - & - & - & - & W & 1 \\
\hline 2 & 2,2 & MAF & - & * & $\mathrm{F}$ & - & - & - & 1 \\
\hline 3 & 2,3 & MAF & - & * & W & - & - & - & 1 \\
\hline 4 & 2,8 & WAF & $\mathrm{F}$ & $\mathrm{F}$ & - & - & - & - & 2 \\
\hline 5 & 3,2 & MAF & W & $S$ & W & W & W & W & 6 \\
\hline 6 & 3,2 & MAF & - & * & - & $\mathrm{F}$ & $\mathrm{F}$ & - & 2 \\
\hline 7 & 3,3 & WAF & - & * & $\mathrm{F}$ & - & - & - & 1 \\
\hline 8 & 3,7 & ND & $S$ & * & - & $\mathrm{F}$ & - & - & 2 \\
\hline 9 & 3,8 & ND & - & - & - & $\mathrm{F}$ & - & - & 1 \\
\hline 10 & 3,9 & ND & - & - & W & $\mathrm{F}$ & - & - & 2 \\
\hline 11 & 4,0 & WAF & $S$ & $S$ & W & $\mathrm{F}$ & W & - & 5 \\
\hline 12 & 4,2 & WAF & W & $M$ & - & - & - & - & 2 \\
\hline 13 & 4,5 & WAF & $\mathrm{F}$ & $\mathrm{F}$ & - & - & - & - & 2 \\
\hline 14 & 4,6 & MAF & - & - & $\mathrm{F}$ & - & - & - & 1 \\
\hline 15 & 5,3 & WAF & $M$ & $M$ & W & $\mathrm{F}$ & - & - & 4 \\
\hline 16 & 5,5 & WAF & - & - & - & $F$ & - & - & 1 \\
\hline 17 & 5,7 & MAF & $S$ & $S$ & W & W & - & $\mathrm{F}$ & 5 \\
\hline 18 & 6,6 & MAF & $\mathrm{F}$ & * & - & - & - & - & 1 \\
\hline 19 & 7,0 & ND & - & - & W & $\mathrm{F}$ & - & - & 2 \\
\hline 20 & 7,2 & WAF & $S$ & * & W & $M$ & w & $M$ & 5 \\
\hline 21 & 7,4 & WAF & - & - & - & $F$ & - & - & 1 \\
\hline 22 & 7,6 & MAF & - & - & - & $F$ & - & - & 1 \\
\hline 23 & 9,1 & ND & - & - & - & - & - & W & 1 \\
\hline 24 & 9,6 & ND & * & $M$ & W & $\mathrm{F}$ & - & - & 3 \\
\hline 25 & 20,0 & ND & - & - & - & $\mathrm{F}$ & - & - & 1 \\
\hline 26 & 20,0 & WAF & $\mathrm{F}$ & - & - & $\mathrm{F}$ & - & - & 2 \\
\hline 27 & 20,0 & WAF & - & - & W & - & - & - & 1 \\
\hline 28 & 20,0 & WAF & W & $S$ & W & $\mathrm{F}$ & W & - & 5 \\
\hline 29 & 20,0 & WAF & W & $M$ & $\mathrm{~F}$ & $F$ & - & W & 5 \\
\hline 30 & 20,0 & WAF & - & - & $\mathrm{F}$ & $F$ & - & - & 2 \\
\hline 31 & 20,0 & ND & $\mathrm{F}$ & - & - & - & - & - & 1 \\
\hline 32 & 20,0 & ND & $\mathrm{F}$ & - & - & $\mathrm{F}$ & - & - & 2 \\
\hline 33 & 20,0 & WAF & - & - & - & $\mathrm{F}$ & - & - & 1 \\
\hline 34 & 20,0 & MAF & - & * & - & - & - & w & 1 \\
\hline 35 & 20,0 & ND & - & * & $\mathrm{F}$ & - & - & - & 1 \\
\hline 36 & 20,0 & MAF & $\mathrm{F}$ & * & - & - & - & - & 1 \\
\hline 37 & 20,0 & WAF & - & * & W & - & - & - & 1 \\
\hline 38 & 20,0 & WAF & - & * & $\mathrm{F}$ & - & - & - & 1 \\
\hline 39 & 20,0 & WAF & - & * & $\mathrm{F}$ & - & - & - & 1 \\
\hline 40 & 20,0 & MAF & - & - & - & $\mathrm{F}$ & - & - & 1 \\
\hline 41 & 20,0 & WAF & - & $M$ & W & W & $w$ & - & 4 \\
\hline
\end{tabular}


Table 2: False positive Pv-pLDH lines in 42 P. falciparum samples with parasite densities $\geq 2 \%$ (Continued)

\begin{tabular}{|c|c|c|c|c|c|c|c|}
\hline WAF & - & * & - & - & $\mathrm{F}$ & - & 1 \\
\hline $\begin{array}{c}\text { Total false positive Pv- } \\
\text { pLDH lines }\end{array}$ & 16 & 11 & 21 & 23 & 7 & 7 & 85 \\
\hline
\end{tabular}

were excluded by PCR analysis. Frequencies for individual brands ranged from $8.2 \%$ (7/85 samples) to $29.1 \%$ (23/ 79 samples).

Plasmodium vivax accounts for almost half of the malaria infections worldwide and is no longer considered as a mild infection: complicated infections have been demonstrated in both endemic countries and in returned travelers $[11,12]$. In addition, $P$. vivax malaria may be chloroquine resistant and has a tendency to relapse [13]. To eradicate the dormant liver stages, primaquine treatment is needed. Primaquine is contraindicated in case of glucose-6-phosphate dehydrogenase (G6PD) deficiency, due to the risk of hemolysis $[14,15]$. G6PD deficiency is common in most $P$. vivax malaria areas; moreover, in these areas G6PD testing is impractical due to a lack of funds, equipments or expertise [16].

RDTs detecting $P$. vivax-specific $\mathrm{pLDH}$ are of additional value for the diagnosis of malaria in both $P$. vivax endemic areas and in the setting of travel medicine. The conventional three-band RDTs detecting a P. falciparumspecific antigen and a pan-Plasmodium antigen cannot distinguish between a $P$. falciparum infection and a mixed infection with $P$. vivax when both test lines are observed. Differentiation between the non-falciparum species is neither possible. Three- or four-band RDTs that target Pv-pLDH have the advantage that they can $\operatorname{detect} P$. vivax in mixed infections: they are an adjunct to microscopy as P. vivax is often microscopically under diagnosed in mixed infections [13]. In addition they can be used to distinguish between Plasmodium ovale and P. vivax [2]. This is an advantage in the non-endemic setting, where microscopic differentiation between $P$. ovale and $P$. vivax is notoriously difficult [17].

Plasmodium vivax-specific RDTs have hardly been evaluated [18-22], and are not discussed in any of the recent reviews on malaria $\operatorname{RDTs}[1,8,17,23]$. The present findings are of concern particularly in areas where $P$. falciparum and $P$. vivax are co-circulating: in case of a twoband Pv-pLDH test, the diagnosis of life-threatening $P$. falciparum malaria may be missed, which is especially of concern in remote areas among less experienced staff and without backup of microscopy. In case of a three- or fourband RDT, the patient may be treated incorrectly with primaquine, leading to severe hemolysis in patients with G6PD-deficiency.

Among the limitations of the present study, we should mention its retrospective nature (precluding further work-up of samples), the test conditions in a reference laboratory (which are more favorable than those in field settings) and the fact that all but one P. falciparum samples were obtained in travelers returning from Africa. In addition, the present study explored false positive $\mathrm{Pv}$ -

Table 3: Numbers of $P$. falciparum samples generating false positive Pv-pLDH lines according to geographic origin

\begin{tabular}{|c|c|c|c|}
\hline \multirow[b]{2}{*}{ Origin (region) } & \multicolumn{3}{|c|}{ Numbers of samples tested* } \\
\hline & Total & Negative & $\begin{array}{c}\text { Positive }^{* *} \text { (\% of total } \\
\text { samples) }\end{array}$ \\
\hline East Africa & 5 & 5 & 0 \\
\hline Middle Africa & 22 & 10 & $12(54.5)$ \\
\hline Southern Africa & 4 & 4 & 0 \\
\hline West Africa & 34 & 14 & $20(58.8)$ \\
\hline West Asia & 1 & 1 & 0 \\
\hline No data & 19 & 9 & $10(52.6)$ \\
\hline All samples & 85 & 43 & $42(49.4)$ \\
\hline
\end{tabular}

* Nine RDT brands were tested with a panel of 85 samples

** Defined as a sample with a false positive Pv-pLDH line in $\geq 1$ RDT brand 
pLDH lines exclusively in samples with high $P$. falciparum parasite densities, in line with the previous observations [2-4]. However, the inclusion of P. falciparum samples of lower parasite density and Plasmodium negative samples would have completed the picture. A prospective study in an area with $P$. falciparum and $P$. vivax coexistence should be performed to assess the relevance of the false positive $\mathrm{Pv}$-pLDH lines in a field setting.

False-positive reactions of $P$. vivax samples with the HRP-2 line and the Pf-pLDH line have been described previously [24-26], but the presently observed false-positive $\mathrm{Pv}$-pLDH lines in P. falciparum samples have only been reported anecdotally [2-4,22]. Among the panel of RDTs tested in the $\mathrm{WHO}$ and Foundation for Innovative New Diagnostics (FIND) study, there were two RDTs with a Pv-pLDH line: according to the tables, one of them generated false positive $\mathrm{Pv}$-pLDH lines in 1.9\% (6/316) of the $P$. falciparum samples tested.

Without exact knowledge of the antibodies targeting the Pv-pLDH antigen, it is difficult to explain the phenomenon, especially since false positive Pv-pLDH lines in the present study occurred not consistently among the different samples and brands. Weak cross-reactions may also be facilitated by slight differences in the composition of the nitrocellulose membrane and the diluent. It is known that several companies use the same pLDH antibodies in their RDT brands [27] and this may also be the case for Pv-pLDH: this is reflected by the identical product presentations and similar false positive rates of RDT brands numbers 6 and 7 and numbers 2 and 3 respectively. In the present study, there was no apparent relation between the false positive Pv-pLDH lines and the geographic origin of the infection, but it should be noted that mainly samples from sub-Saharan Africa were included.

The present study also revealed problems in RDT availability and communication with the supplier in the international market, as eight brands were not delivered despite several reminders. Furthermore, two of the RDTs that claimed to be $P$. vivax-specific by their label and name proved to detect pan-pLDH according to their package insert. Similar confusing names have been noted previously, also for the antibodies targeting aldolase [28].

\section{Conclusion}

The occurrence of false positive Pv-pLDH lines in P. falciparum samples with high parasite densities was observed in six out of nine P. vivax-specific RDTs, in two-, threeand four-band RDTs. The false positive Pv-pLDH lines are of concern because the diagnosis of life-threatening $P$. falciparum malaria may be missed (two-band Pv-pLDH RDT), or the patient will be treated incorrectly with primaquine (three- or four-band RDT), which may cause severe hemolysis in patients with G6PD-deficiency. A prospective study in an area with $P$. falciparum and $P$. vivax coexistence should be performed to assess the relevance of the false positive Pv-pLDH lines in a field setting.

\section{List of abbreviations}

FIND: Foundation of New Innovative Diagnostics; G6PD: Glucose-6-phosphate dehydrogenase; HRP-2: Histidinerich protein 2; ITM: Institute of Tropical Medicine; P: Plasmodium; pan-pLDH: pan Plasmodium-specific parasite lactate dehydrogenase; PCR: Polymerase chain reaction; Pf-pLDH: Plasmodium falciparum-specific parasite lactate dehydrogenase; pLDH: Plasmodium-specific parasite lactate dehydrogenase; Pv-pLDH: Plasmodium vivax-specific parasite lactate dehydrogenase; RDT(s): Rapid diagnostic test(s); WHO: World Health Organization.

\section{Competing interests}

The authors declare that they have no competing interests.

\section{Authors' contributions}

PG and JJ designed the study protocol. MvE and JvdE organized prospective sample collection. JM and PG carried out the test evaluations, LC performed PCR analysis. JM, PG and JJ analyzed and interpreted the results and JM and JJ drafted the manuscript. JM and PG performed statistical analysis. All authors read and approved the final manuscript.

\section{Acknowledgements}

We would like to thank the staff of the Central Laboratory of Clinical Biology (ITM, Antwerp) for technical support.

\section{Author Details}

${ }^{1}$ Faculty of Health, Medicine and Life Sciences (FHML), Maastricht, The Netherlands and 2Department of Clinical Sciences, Institute of Tropical Medicine (ITM), Unit of Tropical Laboratory Medicine, Nationalestraat 155, B 2000 Antwerp, Belgium

Received: 22 March 2010 Accepted: 10 July 2010

Published: 10 July 2010

\section{References}

1. Murray CK, Gasser RA Jr, Magill AJ, Miller RS: Update on rapid diagnostic testing for malaria. Clin Microbiol Rev 2008, 21(1):97-110.

2. Gillet $P$, Bosselaers $K$, Cnops L, Bottieau E, Van Esbroeck M, Jacobs J: Evaluation of the SD FK70 malaria Ag Plasmodium vivax rapid diagnostic test in a non-endemic setting. Malar J 2009, 8:129.

3. Gillet P, van Dijk DP, Bottieau E, Cnops L, Van Esbroeck M, Jacobs J: Test characteristics of the SD FK80 Plasmodium falciparum/Plasmodium vivax malaria rapid diagnostic test in a non-endemic setting. Malar J 2009, 8:262.

4. van Dijk DP, Gillet P, Vlieghe E, Cnops L, van Esbroeck M, Jacobs J: Evaluation of the Palutop +4 malaria rapid diagnostic test in a nonendemic setting. Malar J 2009, 8:293.

5. Composition of macro geographical (continental) regions, geographical sub-regions, and selected economic and other groupings [http://unstats.un.org/unsd/methods/m49/m49regin.htm]

6. Van der Palen M, Gillet P, Bottieau E, Cnops L, Van Esbroeck M, Jacobs J: Test characteristics of two rapid antigen detection tests (SD FK50 and SD FK60) for the diagnosis of malaria in returned travellers. Malar J 2009, 8:90.

7. Gillet P, Mori M, Van Esbroeck M, Van den Ende J, Jacobs J: Assessment of the prozone effect in malaria rapid diagnostic tests. Malar J 2009, 8:271.

8. Moody A: Rapid diagnostic tests for malaria parasites. Clin Microbio/ Rev 2002, 15(1):66-78

9. WHO: List of known commercially-available antigen-detecting malaria RDTs: information for national public health services and UN agencies wishing to procure RDTs. 
10. Chiodini PL, Bowers K, Jorgensen P, Barnwell JW, Grady KK, Luchavez J, Moody AH, Cenizal A, Bell D: The heat stability of Plasmodium lactate dehydrogenase-based and histidine-rich protein 2-based malaria rapid diagnostic tests. Trans R Soc Trop Med Hyg 2007, 101(4):331-337.

11. Habib AG, Singh KS: Respiratory distress in nonimmune adults with imported malaria. Infection 2004, 32(6):356-359.

12. Rogerson SJ, Carter R: Severe vivax malaria: newly recognised or rediscovered. PLoS Med 2008, 5(6):e136

13. Sina B: Focus on Plasmodium vivax. Trends Parasitol 2002, 18(7):287-289.

14. Beutler E, Duparc S: Glucose-6-phosphate dehydrogenase deficiency and antimalarial drug development. Am J Trop Med Hyg 2007, 77(4):779-789.

15. WHO: Guidelines for the treatment of malaria. Geneva: World Health Organization; 2006.

16. Leslie T, Mayan I, Mohammed N, Erasmus P, Kolaczinski J, Whitty CJ, Rowland M: A randomised trial of an eight-week, once weekly primaquine regimen to prevent relapse of plasmodium vivax in Northwest Frontier Province, Pakistan. PLoS One 2008, 3(8):e2861

17. Wongsrichanalai C, Barcus MJ, Muth S, Sutamihardja A, Wernsdorfer WH: A review of malaria diagnostic tools: microscopy and rapid diagnostic test (RDT). Am J Trop Med Hyg 2007, 77(6 Suppl):1 19-127.

18. Sharew B, Legesse M, Animut A, Jima D, Medhin G, Erko B: Evaluation of the performance of CareStart Malaria Pf/Pv Combo and Paracheck Pf tests for the diagnosis of malaria in Wondo Genet, southern Ethiopia. Acta Trop 2009, 111(3):321-324.

19. Mekonnen Z, Ali S, Belay G, Suleman S, Chatterjee S: Evaluation of the performance of CareStart Malaria Pf/Pv Combo rapid diagnostic test for the diagnosis of malaria in Jimma, southwestern Ethiopia. Acta Trop 2010, 113(3):285-288

20. Rakotonirina $H$, Barnadas $C$, Raherijafy $R$, Andrianantenaina $H$, Ratsimbasoa A, Randrianasolo L, Jahevitra M, Andriantsoanirina V, Menard D: Accuracy and reliability of malaria diagnostic techniques for guiding febrile outpatient treatment in malaria-endemic countries. Am J Trop Med Hyg 2008, 78(2):217-221.

21. Kim SH, Nam MH, Roh KH, Park HC, Nam DH, Park GH, Han ET, Klein TA, Lim CS: Evaluation of a rapid diagnostic test specific for Plasmodium vivax. Trop Med Int Health 2008, 13(12):1495-1500.

22. WHO: Malaria Rapid Diagnostic Test Performances. World Health Organization; 2009 .

23. Marx A, Pewsner D, Egger M, Nuesch R, Bucher HC, Genton B, Hatz C, Juni $P$ : Meta-analysis: accuracy of rapid tests for malaria in travelers returning from endemic areas. Ann Intern Med 2005, 142(10):836-846.

24. Pieroni P, Mills CD, Ohrt C, Harrington MA, Kain KC: Comparison of the ParaSight-F test and the ICT Malaria Pf test with the polymerase chain reaction for the diagnosis of Plasmodium falciparum malaria in travellers. Trans R Soc Trop Med Hyg 1998, 92(2):166-169.

25. Cho D, Kim KH, Park SC, Kim YK, Lee KN, Lim CS: Evaluation of rapid immunocapture assays for diagnosis of Plasmodium vivax in Korea. Parasitol Res 2001, 87(6):445-448

26. Soto Tarazona A, Solari Zerpa L, Mendoza Requena D, Llanos-Cuentas A, Magill A: Evaluation of the rapid diagnostic test OptiMAL for diagnosis of malaria due to Plasmodium vivax. Braz J Infect Dis 2004, 8(2):151-155.

27. Makler MT, Piper RC: Rapid malaria tests: where do we go after 20 years? Am J Trop Med Hyg 2009, 81(6):921-926.

28. Mason DP, Kawamoto F, Lin K, Laoboonchai A, Wongsrichanalai C: A comparison of two rapid field immunochromatographic tests to expert microscopy in the diagnosis of malaria. Acta Trop 2002, 82(1):51-59.

doi: $10.1186 / 1475-2875-9-198$

Cite this article as: Maltha et al., Malaria rapid diagnostic tests: Plasmodium falciparum infections with high parasite densities may generate false positive Plasmodium vivax pLDH lines Malaria Journal 2010, 9:198

\section{Submit your next manuscript to BioMed Central and take full advantage of:}

- Convenient online submission

- Thorough peer review

- No space constraints or color figure charges

- Immediate publication on acceptance

- Inclusion in PubMed, CAS, Scopus and Google Scholar

- Research which is freely available for redistribution

Submit your manuscript at www.biomedcentral.com/submit
C Biomed Central 